\title{
Primary Nucleation Rate and Radial Growth Rate of Poly(ethylene oxide) Spherulite in Viscous Solutions
}

\author{
Takashi SASAKI, ${ }^{\dagger}$ Yasuhisa YAMAMOTO, and Toshisada TAKAHASHI
}

Department of Materials Science \& Engineering, Fukui University, Fukui 910-8507, Japan

(Received August 23, 1999)

\begin{abstract}
The primary nucleation rate $I$ and radial growth rate $G$ of the isothermal spherulitic growth of poly(ethylene oxide) in tripropionin solutions of 5, 10, and $30 \mathrm{wt} \%$ polymer concentrations were studied by direct in situ observation using a CCD camera. $I$ was found to decrease with pre-annealing at $200^{\circ} \mathrm{C}$ up to $c a .200 \mathrm{~h}$, while $G$ was almost constant. These results suggest that there exist micro-residuals of a crystalline-like structure that act as dominant seeds for primary nucleation and that they become dissolved with structural relaxation during pre-annealing, resulting in the solution approaching a homogeneous state. The supercooling dependencies of $I$ and $G$ were independently investigated, and it was found that the fold surface free energy and activation energy of the polymer diffusion increase with decreases in the polymer content of the solution. The frictional interaction between the polymer and solvent is also discussed on the basis of the reaction rate theory of Kramers.
\end{abstract}

KEY WORDS Poly(ethylene oxide)/Spherulitic Growth in Solution / Primary Nucleation Rate / Radial Growth Rate / Surface Free Energy / Diffusion Limit / Morphology /

Crystallization of polymers in solutions includes essentially complicated phenomena. These phenomena are affected by the solution properties (structure and dynamics) in a non-equilibrium supercooled state as well as by the phase separation phenomenon, and their kinetics is not fully understood. It has been found that, in general, both the crystallization rate and the crystalline morphology are strongly dependent on the solvent, ${ }^{1}$ indicating that the polymer-solvent interaction in the supercooled state plays an essential role in both the kinetics and thermodynamics of crystallization. At the present stage, it is of great importance to elucidate how the polymer-solvent interaction influences both the initial primary nucleation and the growth behavior in solution crystallization.

Our previous study on the spherulitic growth of poly(ethylene oxide) (PEO) in a viscous solution of tripropionin (glycerol tri- $n$-propionate, referred to as TP henceforth) has shown that pre-annealing for dissolution at $200^{\circ} \mathrm{C}$ considerably slows the total crystallization rate. ${ }^{2}$ This indicates that certain structural relaxation proceeds with the dissolution, and this is very sensitive to the crystallization behavior. When the pre-annealing for dissolution is not sufficient (in terms of time or temperature), the solution is not in an equilibrium state. In such a heterogeneous solution, primary nucleation can easily occur because micro-residuals of a crystalline-like structure remain in the solution, leading to a higher total crystallization rate. As structural relaxation proceeds over a long annealing time at a high temperature, the micro-residuals are dissolved and annihilated, and finally, a sufficiently homogenous structure is attained in which the primary nucleation rate reaches its asymptotic low value. If the above consideration is valid, annealing treatment suppresses mainly the primary nucleation rate.

We have also found that for spherulitic growth in a PEO-TP solution at a high concentration (30 wt\%), the apparent activation energy of the diffusion factor is lower than that of the viscosity of TP. ${ }^{2}$ This indicates that the diffusion aspect belongs to the low-friction limit of the reaction rate theory of Kramers, ${ }^{3,4}$ i.e., the frictional interaction between the polymer and solvent is extremely low. The same feature has also been found in solution crystallization of an isotactic polystyrene-TP system, ${ }^{5}$ and this may be an unique character intrinsic to a solvent with high viscosity such as TP. ${ }^{2,6,7}$

In the present study, we investigated the primary nucleation rate and radial growth rate of spherulitic growth in a PEO-TP system in order to elucidate their behavior with pre-annealing as well as their supercooling dependencies at three PEO concentrations $(5,10$, and $30 \mathrm{wt} \%$ ). We then evaluated the surface free energy parameters and activation energy for the diffusion of PEO molecules. Based on the results, discussion on the nucleation process, polymer-solvent frictional interaction, and morphological change with supercooling is presented.

\section{EXPERIMENTAL}

\section{Solution Preparation and Measurements}

Commercial PEO $\left(M_{n}=2.04 \times 10^{4}, M_{w}=2.16 \times 10^{4}\right.$, $M_{w} / M_{n}=1.06$ ) was purchased from Scientific Polymer Products, Inc. TP (Tokyo Kasei Co.) was distilled under vacuum and was filtered using a $0.2-\mu \mathrm{m}$ mesh Millipore filter just before use. In a dust-free atmosphere (class $100)$, PEO was added to TP to make 5, 10, and $30 \mathrm{wt} \%$ (PEO content) mixtures. These were degassed and sealed under vacuum $\left(<10^{-5} \mathrm{mmHg}\right)$ with several pumpand-thaw cycles in a quartz cell of 1 or $10 \mathrm{~mm}$ in thickness, and then the pre-annealing for dissolution was executed at $200^{\circ} \mathrm{C}$.

The isothermal spherulitic growth in the above degassed solutions sealed in cells was observed using a CCD camera (Flovel HCC-600) equipped with a zoom lens (Navitar Macro Zoom), after various pre-annealing times. The image data were recorded by a videotape recorder. The crystallization temperature was fixed to 30 , 33 , and $35^{\circ} \mathrm{C}$ for 5,10 , and $30 \mathrm{wt} \%$ solutions, respec- 


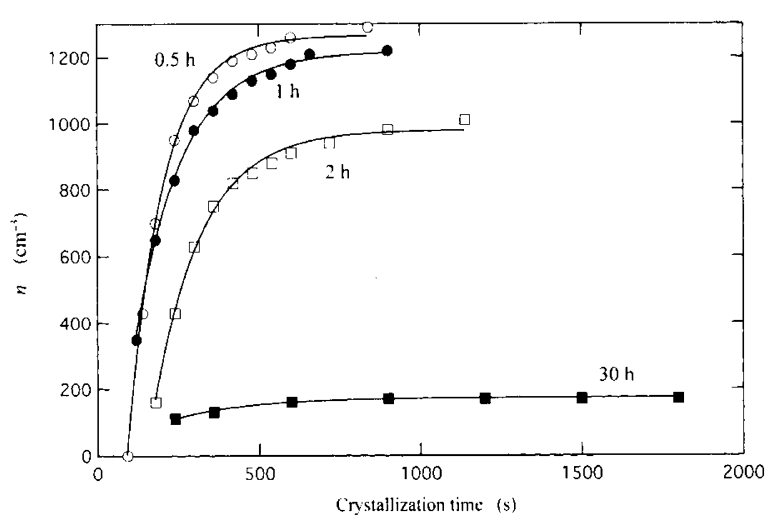

Figure 1. Typical profiles of the number density of spherulites $n$ with respect to crystallization time in $10 \mathrm{wt} \% \mathrm{PEO}-\mathrm{TP}$ solution after various pre-annealing times as indicated in the figure. The crystallization temperature is $33^{\circ} \mathrm{C}$. The solid lines indicate the fitted curves of eq 2 by the non-linear least squares method.

tively. Temperature was kept within $\pm 0.02^{\circ} \mathrm{C}$ by a thermo-regulated water bath controlled by a Taitec DG10. The primary nucleation rate $I$ was evaluated by counting the number of spherulites in a fixed area at different crystallization times. The radial spherulitic growth rate $G$ was evaluated by measuring the time variation in maximum diameter of an arbitrary selected spherulite. Such measurements of $G$ were done for several spherulites, and their average was taken as the experimental value of $G$. We also investigated the crystallization temperature (supercooling) dependencies of $I$ and $G$ for solution pre-annealed for $200 \mathrm{~h}$ at $200^{\circ} \mathrm{C}$ in the range $\Delta T=14-44 \mathrm{~K}$. In these measurements, the sample solutions were re-annealed for dissolution at $70^{\circ} \mathrm{C}$ for $8 \mathrm{~min}$ and were quenched to various crystallization temperatures.

\section{Determination of the Equilibrium Dissolution Tempera- ture}

We prepared several crystallized PEO samples of different lamellar thicknesses by a self-seeding method established for PEO :8,9 the PEO as-received sample was (1) heated at $93.6^{\circ} \mathrm{C}\left(=T_{\mathrm{m}}+30 \mathrm{~K}\right.$, where $T_{\mathrm{m}}$ was determined for the as-received PEO by a DSC heating scan at $0.5^{\circ} \mathrm{C} \mathrm{min}{ }^{-1}$ ) for $10 \mathrm{~min}$, (2) quenched to $40^{\circ} \mathrm{C}$, (3) held for $20 \mathrm{~min}$, (4) again heated to $63.1^{\circ} \mathrm{C}$ (self-seeding temperature) at a rate $0.1^{\circ} \mathrm{C} \min ^{-1},(5)$ held for $20 \mathrm{~min}$, and finally (6) cooled rapidly to various crystallization temperatures. All these procedures were done in a DSC apparatus Perkin Elmer DSC 7. The lamellar thicknesses of these samples were determined by electron microscopy, as described below.

We executed the etching procedure for the above crystallized PEOs according to Cheng et al. ${ }^{10}$ in a $\mathrm{C}_{2} \mathrm{H}_{5} \mathrm{ONa}$ / $\mathrm{C}_{2} \mathrm{H}_{5} \mathrm{OH}\left(21 / 79\right.$ weight fraction) mixture at $25^{\circ} \mathrm{C}$, and we washed them with $\mathrm{C}_{2} \mathrm{H}_{5} \mathrm{OH}$ at the same temperature. The etched specimens were obliquely shadowed with Pt$\mathrm{Pd}$ by vacuum evaporation, followed by carbon coating, and then the carbon replicas were separated in water. Observation was done using a transmission electron microscope Jeol JEM-2000 FXII, and the lamellar thickness of each specimen was estimated from the average width of the shadows of the observed lamellae. To determine the dissolution temperature $T_{\mathrm{d}}$ in TP, DSC heating scans were executed at a rate of $0.5^{\circ} \mathrm{C} \mathrm{min}{ }^{-1}$ for PEO-TP mixtures $(5,10$, and $30 \mathrm{wt} \%$ of $\mathrm{PEO})$ in aluminum sealed pans.

Finally, we determined the equilibrium dissolution temperature $T_{\mathrm{d}}{ }^{\circ}$ from the obtained data on the following relationship :

$$
T_{\mathrm{d}}=T_{\mathrm{d}}^{\circ}-\frac{2 T_{\mathrm{m}}^{\circ} \sigma_{\mathrm{e}}}{\Delta H_{\mathrm{m}} l}
$$

where $T_{\mathrm{m}}{ }^{\circ}$ is the equilibrium melting temperature, $\sigma_{\mathrm{e}}$ is the fold surface free energy (for the above self-seeded melt crystallization), $\Delta H_{\mathrm{m}}$ is the heat of fusion, and $l$ is the lamellar thickness. The obtained equilibrium dissolution temperatures are $322.3,322.2$, and $322.0 \mathrm{~K}$ for 5 , 10 , and $30 \mathrm{wt} \%$ solutions, respectively, and these values were employed in the data analysis.

\section{Measurement of the Heat of Dissolution}

In order to estimate the surface free energies, we determined the heat of dissolution $\Delta H_{\mathrm{d}}$ for the present PEO-TP system by DSC measurements. As-received PEO and TP mixtures of 5, 10, and $30 \mathrm{wt} \%$ (PEO content) were put into aluminum sealed pans, and heating scans were executed at $2^{\circ} \mathrm{C} \mathrm{min}^{-1}$ by a Perkin Elmer DSC 7. We evaluated the heat of dissolution by integrating the detected endotherms. The obtained values were corrected by taking into account the degree of crystallization of the as-received $\mathrm{PEO}$, which was determined by density measurement.

\section{RESULTS AND DISCUSSION}

\section{Pre-annealing Effect}

Figure 1 shows typical profiles of the time-evolution of the number density of spherulites $n$ during isothermal crystallization. The non-linearity observed in Figure 1 suggests a heterogeneous nature of the primary nucleation event. In addition, it should also be considered that the decrease in the PEO content in the liquid phase during isothermal crystallization as the phase separation proceeds also slows the primary nucleation rate. We analyzed the experimental data with the empirical equation

$$
n(t)=n_{\mathrm{f}}\left\{1-\exp \left[-c\left(t-t_{0}\right)\right]\right\}
$$

by the non-linear least squares fitting. From the results, we obtained the values of the primary nucleation rate $I$, the ultimate spherulite density $n_{\mathrm{f}}$ (asymptotic final value of the number density), and the induction period $t_{0}$. Here, we define the primary nucleation rate $I$ as the absolute value of the initial slope of the $n v s$. $t$ curve, i.e., $I=n_{\mathrm{f}} c$.

Figure 2 shows the variation in $I$ with respect to preannealing time. The figure clearly shows that $I$ decreases with pre-annealing time up to $c a .200 \mathrm{~h}$. We previously confirmed by viscometry and infrared spectroscopy that degradation or depolymerization is scarcely detected even after $200 \mathrm{~h}$ of annealing at $200^{\circ} \mathrm{C}{ }^{2}$ Hence, the present decrease in $I$ is attributed to the structural relaxation of the solution approaching an equilibrium homogeneous state. The fact that this decrease in $I$ is more remarkable at higher concentrations supports the 


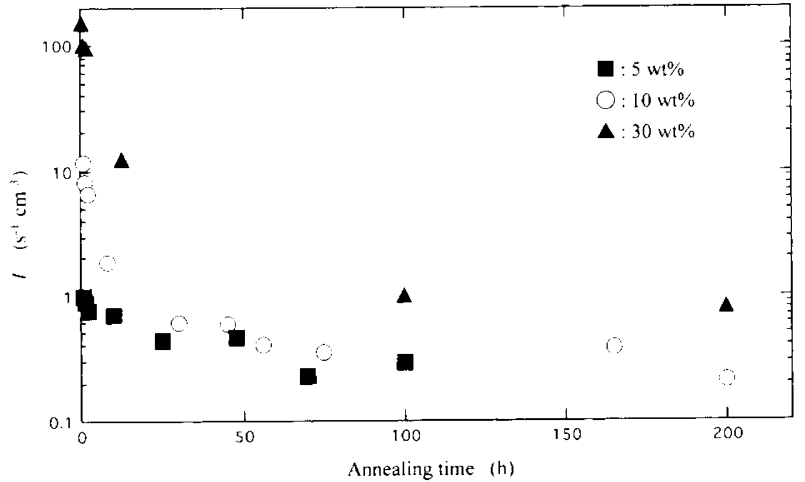

Figure 2. Primary nucleation rate $I$ with respect to preannealing time $\left(200^{\circ} \mathrm{C}\right)$. The crystallization temperatures are 30 , 33 , and $35^{\circ} \mathrm{C}$ for 5,10 , and $30 \mathrm{wt} \%$ solutions, respectively.

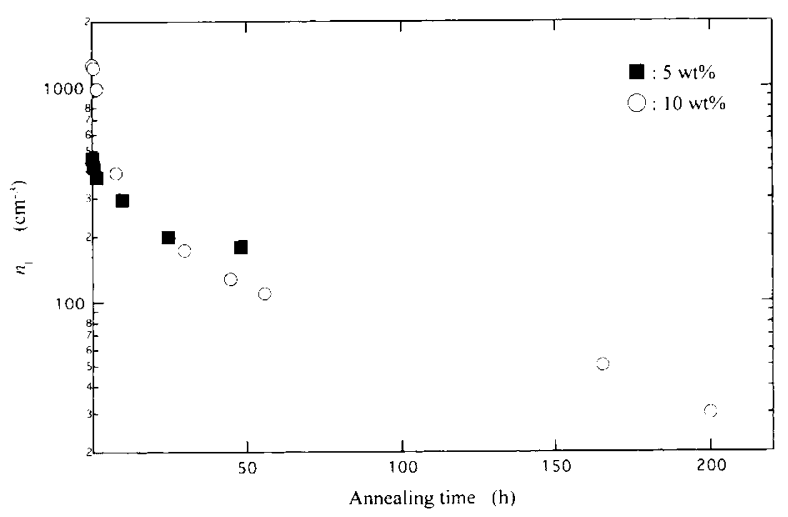

Figure 3. Ultimate spherulite density $n_{\mathrm{f}}$ with respect to preannealing time. The crystallization temperatures are the same as those in Figure 2.

above speculation, because it is difficult to obtain a homogeneous solution at high PEO concentrations. Figure 3 shows changes in $n_{\mathrm{f}}$ with respect to pre-annealing time. It can be seen that $n_{\mathrm{f}}$ also decreases with pre-annealing time, indicating that the number of heterogeneous sites where primary nucleation can take place decreases as pre-annealing proceeds. The above results indicate that the primary nucleation of the present system is a kind of heterogeneous nucleation; i.e., the seed for primary nucleation consists of a small portion of PEO molecules which are not fully dissolved and aggregate in a certain crystalline-like structure or conformation.

Figure 4 shows typical time variations in the diameter of growing spherulites. We can see that the spherulites grow linearly with time, in contrast to the number density of spherulites. This suggests that the spherulitic growth rate $G$, compared with $I$, is rather insensitive to the decrease in PEO concentration during crystallization. The present measurements were done in the initial stage of crystallization because of the experimental limitation : counting the number and measuring the diameter $d$ of spherulites are possible only when they are isolated with no overlapping that occurred in the later stage. In practice, the time range of the present rate

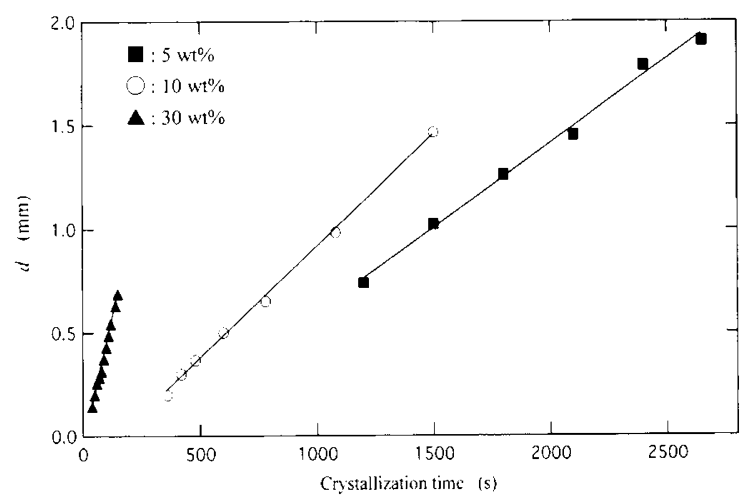

Figure 4. Time variations of the diameter $d$ of the growing PEO spherulites in the pre-annealed TP solutions. The annealing times are 48,56 , and $50 \mathrm{~h}$ for 5,10 , and $30 \mathrm{wt} \%$ solutions, respectively. The crystallization temperatures are the same as those in Figure 2.

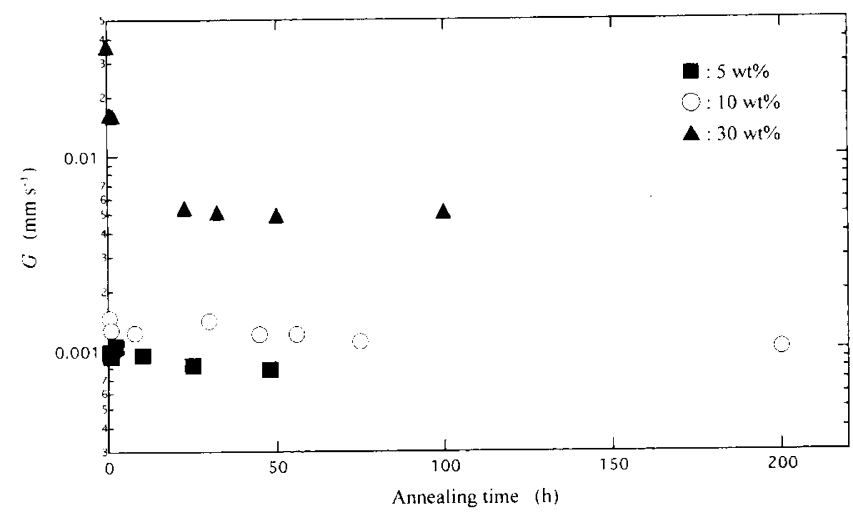

Figure 5. Radial spherulitic growth rate $G$ with respect to preannealing time. The crystallization temperatures are the same as those in Figure 2.

measurements did not exceed $30 \%$ of the total crystallization time in which the crystallinity reaches its final value. Thus, the effect of the decreasing PEO concentration of the liquid phase during crystallization may not be significant for the measurements of $G$.

Figure 5 shows the values of $G$ obtained from the slopes of $d v s$ s $t$ plots with respect to pre-annealing time. As can be seen in this figure, $G$ is almost constant except for the data of $30 \mathrm{wt} \%$ solution in the early annealing time region $(<c a .4 \mathrm{~h})$ where a rapidly decreasing profile is observed. The fact that $G$ is not susceptible to pre-annealing indicates that the decrease in the total crystallization rate with pre-annealing ${ }^{2}$ is mainly due to the decrease in the primary nucleation rate. However, the steep decreasing tendency seen in the initial part for $30 \mathrm{wt} \%$ solution suggests that a rapid structural relaxation in the initial annealing stage impedes even radial growth, i.e., arrival and attachment of PEO chains to the growth surface or surface nucleation. ${ }^{11-13}$

\section{Primary Nucleation Rate}

The above results indicate that the structural relaxation effect in the PEO-TP solutions seems to be saturated after pre-annealing at $200^{\circ} \mathrm{C}$ for $200 \mathrm{~h}$. We there- 


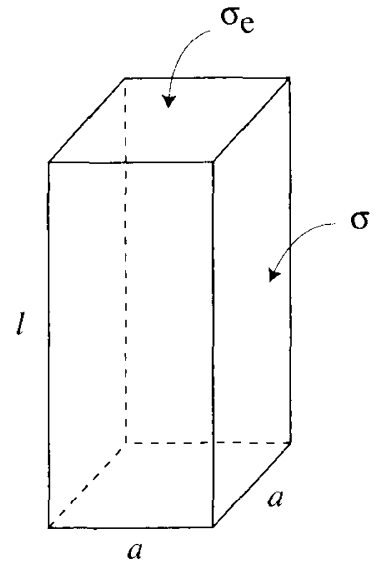

Figure 6. Primary nucleus.

fore investigated the supercooling dependencies of the two rates $I$ and $G$ for solutions pre-annealed under this condition. To treat the nucleation rate in polymer solutions, an entropic contribution to the free energy of nucleation due to separation of polymer from solvent should be taken into account. ${ }^{1,14,15}$ In this case, the free energy of formation of the primary nucleus depicted in Figure 6 can be expressed as :

$$
\Delta \phi=-a^{2} l \Delta f+4 a l \sigma+2 a^{2} \sigma_{e}-k T \frac{a^{2}}{b^{2}} \ln c
$$

where $\Delta f$ is the free energy difference between the amorphous and crystalline states in the polymer-solvent mixture $\left(\Delta f=\Delta H_{\mathrm{d}} \Delta T / T_{\mathrm{d}}{ }^{\circ}\right), \sigma$ is the free energy of the lateral surface, $k$ is the Boltzmann constant, $T$ is the crystallization temperature, $b$ is the thickness of a stem, and $c$ is the volume fraction of the polymer. The last term of the right hand side of eq 3 is derived from the probability of selecting $a^{2} / b^{2}$ chains of length $l$ from the solution. From eq 3 , the critical nucleus sizes $a^{*}$ and $l^{*}$ are derived as

$$
a^{*}=\frac{4 \sigma}{\Delta f}, l^{*}=\frac{4 \sigma_{e}}{\Delta f}-\frac{2 k T}{b^{2} \Delta f} \ln c
$$

and the free energy for this critical nucleus formation is

$$
\Delta \phi^{*}=\frac{32 \sigma^{2} \sigma_{\mathrm{e}}}{(\Delta f)^{2}}-\frac{16 \sigma^{2} k T}{b^{2}(\Delta f)^{2}} \ln c
$$

Thus, the primary nucleation rate $I$ is given by

$$
I=I_{0} \exp \left[-\frac{A\left(T_{\mathrm{d}}{ }^{\circ}\right)^{2}}{T(\Delta T)^{2}}+\frac{\mathrm{B}\left(T_{\mathrm{d}}{ }^{\circ}\right)^{2} \ln c}{(\Delta T)^{2}}\right]
$$

with

$$
A=\frac{32 \sigma^{2} \sigma_{\mathrm{e}}}{k\left(\Delta H_{\mathrm{d}}\right)^{2}}, B=\frac{16 \sigma^{2}}{b^{2}\left(\Delta H_{\mathrm{d}}\right)^{2}}
$$

Since the variation in $T^{-1}$ in the exponent of eq 6 is practically negligible compared with that of $\Delta T^{-2}$ in the present experimental range of $T, I$ can approximately be written as

$$
I=I_{0} \exp \left[-\frac{A^{\prime}\left(T_{\mathrm{d}}{ }^{\circ}\right)^{2}}{(\Delta T)^{2}}\right]
$$

with

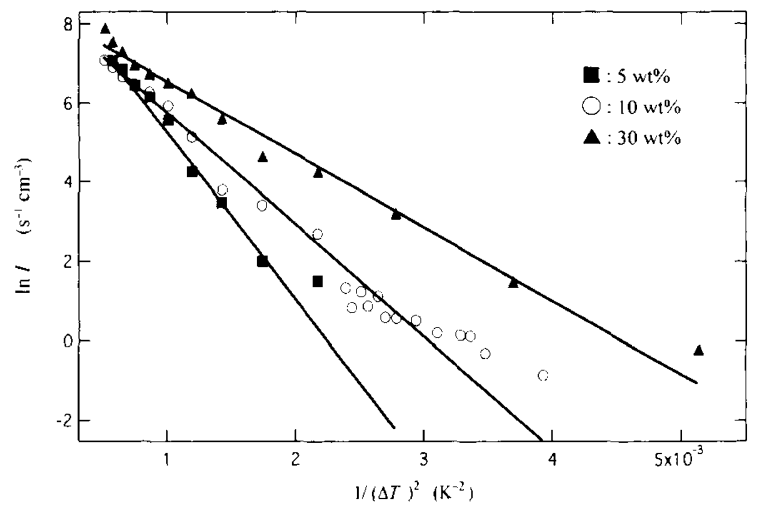

Figure 7. Plots of $\ln I v s . \Delta T^{-2}$ at different PEO concentrations. The solid lines were obtained by the least squares method.

$$
A^{\prime}=\frac{A\left(T_{\mathrm{d}}{ }^{\circ}\right)^{2}}{T_{\mathrm{av}}}-B\left(T_{\mathrm{d}}^{\circ}\right)^{2} \ln c
$$

where $T_{\mathrm{av}}$ is the average crystallization temperature of experiment. Figure 7 shows $\ln I$ plotted against $\Delta T^{-2}$. We obtained best-fit lines from the data in Figure 7 by the least squares method and estimated the values of parameter $A^{\prime}$ from their slopes. Table I lists the results. Apparently, the value of $A^{\prime}$ decreases with increases in the PEO content, which is qualitatively consistent with the $c$ dependence of $A^{\prime}$ seen in eq 8 .

\section{Radial Growth Rate}

Figure 8 shows plots of $G$ vs. $T$ for the pre-annealed solutions. We analyzed the present data on the basis of the surface nucleation theory ${ }^{11-13,16}$ taking into account the diffusion factor. ${ }^{2,5,17}$ Here again, we introduce an extra entropic term characteristic to the crystallization from polymer-solvent mixture in the similar manner to that in the previous sub-section, and the radial growth rate of spherulites are given as ${ }^{15}$

$$
G=G_{0} \exp \left(-\frac{\mathrm{C} T_{\mathrm{d}}{ }^{\circ}}{T \Delta T}+\frac{D T_{\mathrm{d}}{ }^{\circ} \ln c}{\Delta T}\right) \exp \left[-\left(E_{\mathrm{a}}+E_{\eta}\right) / k T\right](9)
$$

with

$$
C=\frac{x b \sigma \sigma_{e}}{k \Delta H_{\mathrm{d}}}, \quad D=\frac{2 \sigma}{b \Delta H_{\mathrm{d}}}
$$

where $E_{\mathrm{a}}$ is the activation energy of polymer diffusion in the radial growth process, $E_{\eta}$ is the activation energy of solvent viscosity, and $x$ is a constant depending on the regime of surface nucleation ( 2 or 4 ). In eq 9 , it is assumed that the diffusion aspect of the crystallization process belongs to the Kramers diffusion limit, i.e., the apparent activation energy is equal to $E_{\mathrm{a}}+E_{\eta} \cdot{ }^{3,18}$ This postulate means that the frictional interaction between the polymer and solvent is extremely large (which is usually the case for an equilibrium solution).

We analyzed the data in Figure 8 by directly fitting the calculated curve of eq 9 to the data by the non-linear least squares method, $G_{0}, C, D$, and $E_{\text {a }}$ being treated as variable parameters. Here, we employed $E_{\eta}=6.5 \mathrm{kcal}$ mol $^{-1}$, which was obtained previously. ${ }^{6}$ The results are shown by solid curves in Figure 8, which indicate that eq 9 can provide good fittings. From the obtained best-fit 


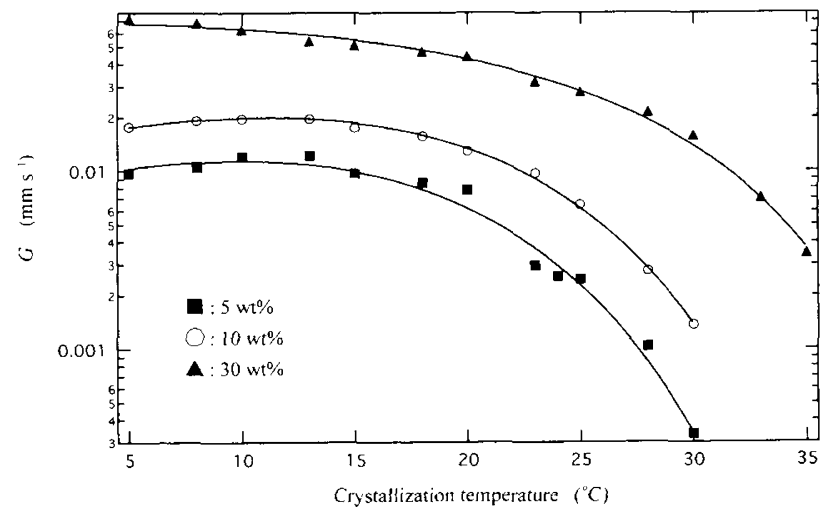

Figure 8. Radial spherulitic growth rate $G$ with respect to crystallization temperature. The solid lines indicate the fitted curves of eq 9 by the non-linear least squares method.

Table I. Heat of dissolution and parameters obtained from the primary nucleation rate and radial growth rate data

\begin{tabular}{lccc}
\hline PEO concentration /wt $\%$ & 5 & 10 & 30 \\
\hline$c$ & 0.0444 & 0.0892 & 0.274 \\
$\Delta H_{\mathrm{d}}\left(10^{9} \mathrm{erg} \mathrm{cm}^{-3}\right)$ & 2.39 & 2.31 & 2.29 \\
Results from primary nucleation rate & & & \\
$A^{\prime}\left(10^{3} \mathrm{~K}^{2}\right)$ & 4.22 & 2.83 & 1.85 \\
Results from radial growth rate & & & \\
$C(\mathrm{~K})$ & 133 & 92.1 & 26.8 \\
$D$ & 0.103 & 0.127 & 0.120 \\
$\sigma\left(\mathrm{erg} \mathrm{cm}^{-2}\right)$ & 5.5 & 6.8 & 6.3 \\
$\sigma_{\mathrm{e}}\left(\mathrm{erg} \mathrm{cm}^{-2}\right)$ & 83 & 47 & 15 \\
$E_{\mathrm{a}}\left(\mathrm{kcal} \mathrm{mol}^{-1}\right)$ & & & \\
Diffusion limit & 17.8 & 14.6 & -1.76 \\
Low-friction limit & 24.3 & 21.1 & 4.74 \\
\hline
\end{tabular}

parameters, we estimated $\sigma$ and $\sigma_{\mathrm{e}}$, assuming that $b=$ $4.63 \AA^{19}$ and $x=2$ (regime II). The results are listed in Table I. We can see that the fold surface free energy $\sigma_{e}$ decreases with increasing $c$, while the lateral surface free energy $\sigma$ shows no apparent dependence on $c$. This may be due to the heterogeneity associated with incomplete dissolution still remaining at higher polymer concentrations even in the pre-annealed solution.

In the above analysis of radial growth rate, we have taken into account the diffusion factor. We have also done the data analysis neglecting the diffusion factor, but in this case, unrealistic negative surface free energies have been obtained. Thus, it is revealed that in the analysis of $G$ with respect to $\Delta T$, we should introduce the diffusion factor. For the primary nucleation rate $I$, however, plots in Figure 7 seem to be linear without taking into account the diffusion factor. This is mainly due to the stronger dependence on $\Delta T$ of $I$ than that of $G$, and the former overcomes the temperature dependence of the diffusion effect in the present temperature range.

The value of the parameter $E_{\text {a }}$ decreases with increases in the PEO concentration (Table I), and for 30 wt $\%$ solution, we have an unrealistic negative value in the case of the diffusion limit. This indicates that the assumption of the diffusion limit is not appropriate for 30 wt\% PEO solution, as was reported in our previous paper. $^{2}$ In the case of the low-friction limit, $E_{\mathrm{a}}$ is $6.5 \mathrm{kcal}$ $\mathrm{mol}^{-1}\left(=E_{\eta}\right)$ higher than in the case of the diffusion limit, as shown in Table I. The value of $4.74 \mathrm{kcal} \mathrm{mol}^{-1}$ (a)

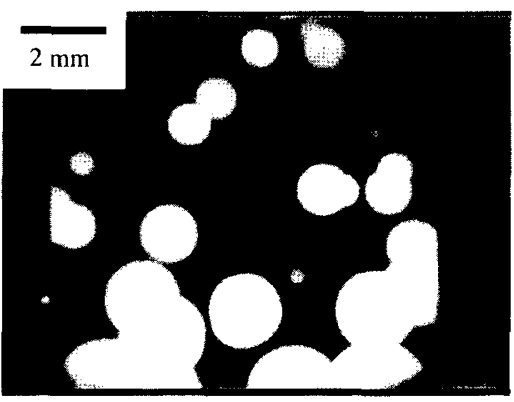

(b)

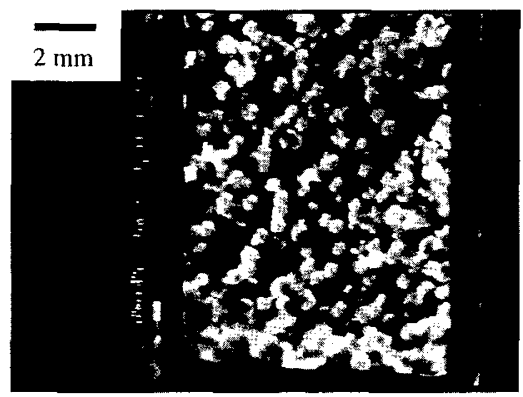

(c)

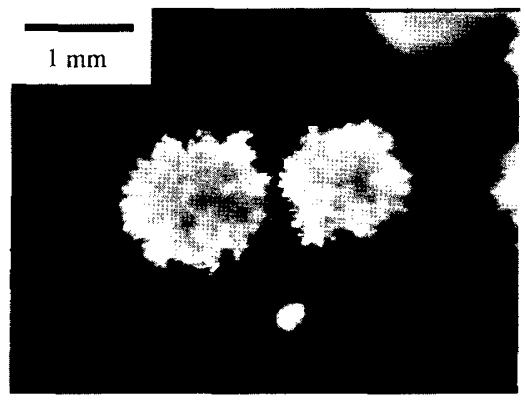

Figure 9. PEO spherulites appeared in $10 \mathrm{wt} \% \mathrm{TP}$ solution at (a) : $\Delta T=24 \mathrm{~K}$, (b) : $\Delta T=19 \mathrm{~K}$, and (c) : $\Delta T=16 \mathrm{~K}$.

for the $30 \mathrm{wt} \%$ solution may be somewhat high even considering the calculated result for the local motions of a PEO chain. ${ }^{20}$ At any rate, the result indicates that the frictional interaction between polymer and solvent is rather weak, and this may be due to the unique feature of viscous solvents with ester groups such as TP, where molecules tend to associate with each other via ester groups and to form a network structure. ${ }^{2}$ Probably in the present PEO-TP system at $30 \mathrm{wt} \%$, PEO chains merely diffuse through the set network of TP with a weak interaction. As for the two lower concentrations, on the other hand, $E_{\mathrm{a}}$ is rather high even when we assume the Kramers diffusion limit. These results suggest that the elementary process of polymer diffusion during spherulitic growth at a low concentration contains large scale motions that require the PEO chain to overcome a high energy barrier. Spherulitic growth in a solution with a low PEO concentration would require aggregation from more highly isolated state of PEO molecules, and in this case, PEO chains must travel through longer paths, and comparatively large scale motions are needed.

\section{Morphology}

In the present PEO-TP system, the spherulites were 
relatively large compared with those grown in the other solvents such as toluene, and $N, N$-dimethylacetamide. This indicates that in the PEO-TP system, the primary nucleation rate is relatively small compared with the radial growth rate. This tendency is dependent on the solvent and, therefore, reflects differences in both the surface free energies of the critical nucleus and the heat of dissolution with respect to the solvent. In addition, the low primary nucleation rate for the PEO-TP system may be partially due to the high viscosity of TP. However, this is not the place to discuss in detail the solvent dependence; such discussion will be presented in a forthcoming paper.

The morphology of a PEO spherulite in a TP solution changes according to both the supercooling and the PEO concentration. Figure 9 shows some typical appearances of spherulites obtained in the $10 \mathrm{wt} \%$ solution. At higher supercoolings, each spherulite has a spherical shape with an apparently smooth surface, as shown in Figure 9a. At lower supercoolings, on the other hand, nonspherical particles with a rather ragged contour or rough surface appear as shown in Figure 9c. These probably consist of a number of aggregated small crystallites (axialites).

The mechanism of the above spherulite-axialite transition according to $\Delta T$ is not clear at the present time. The problem concerns the mechanism of the spherulitic growth of PEO. ${ }^{10,21}$ At higher supercoolings, splitting and branching may occur in the initial precursor lamellae, and also they tend to grow into a multi-layer structure leading to spherulitic morphology, while at lower supercoolings, the precursor lamellae grow into axialites with less splitting and branching. The growth mechanism may be controlled by both the mobility of PEO in the viscous medium and the nucleation rates (primary and surface), and thus, the ragged particles tend to occur when the polymer diffusion rate is relatively high compared with the nucleation rates.

The results of our experiments also revealed that the critical supercooling $\Delta T^{*}$ below which spherulites with a ragged shape are clearly observed decreases with increases in the $\mathrm{PEO}$ concentration; i.e., $\Delta T^{*} \approx 24 \mathrm{~K}$ at 5 $\mathrm{wt} \%, 22 \mathrm{~K}$ at $10 \mathrm{wt} \%$, and $11 \mathrm{~K}$ at $30 \mathrm{wt} \%$. This indicates that the ragged particles are easily formed at low $\mathrm{PEO}$ concentrations, where the mobility of PEO is high and the nucleation rates are low.

\section{CONCLUSIONS}

The present study revealed that the primary nucleation rate of spherulitic growth in $\mathrm{PEO}-\mathrm{TP}$ viscous solutions decreases with pre-annealing time, while the radial growth rate is almost constant except in the initial stage of annealing (within ca. $4 \mathrm{~h}$ ). This result suggests that the heterogeneous sites that promote the primary nucleation tend to be annihilated by annealing at $200^{\circ} \mathrm{C}$ for over ca. $200 \mathrm{~h}$. Also, the concentration dependencies of $I$ and $G$ can be interpreted by introducing the extra entropic terms that are due to separation of polymer from polymer-diluent mixture. However, even for solutions pre-annealed under the above condition, a heterogeneous feature due to incomplete dissolution (or impurities) is still suggested by estimation of the folding surface free energy from the data of $G$ with respect to $\Delta T$.

The present results also showed that the activation energy $E_{\mathrm{a}}$ of polymer diffusion during radial growth decreases with increases in the PEO concentration. This result indicates that the elementary motional modes involved in the $\mathrm{PEO}$ diffusion process vary with the $\mathrm{PEO}$ concentration and that smaller scale motions become dominant at higher PEO concentrations. Also, the frictional interaction between $\mathrm{PEO}$ and TP was found to be extremely weak (Kramers low-friction limit) at $30 \mathrm{wt} \%$. This may reflect the specific character of the viscous solvent TP which possesses ester groups. On the other hand, we could not elucidate the frictional feature at the two lower concentrations ( 5 and $10 \mathrm{wt} \%$ ). More precise experimental data for solutions of low concentration are needed to scrutinize this point.

Acknowledgment. We thank Prof. K. Tsubakiyama, Fukui University, for his kind permission to use a vacuum line apparatus. This work was supported by a Grant-in-Aid for Scientific Research (No. 10750647) from the Ministry of Education, Science, Sports and Culture of Japan.

\section{REFERENCES}

1. L. Mandelkern, "Crystallization of Polymers", McGraw-Hill, New York, N.Y., 1964.

2. T. Sasaki, Y. Yamamoto, and T. Takahashi, Polym. J., 30, 868 (1998).

3. H. A. Kramers, Physica, 7, 284 (1940).

4. P. Hänggi, P. Talkner, and M. Borkovec, Rev. Mod. Phys., 62, 251 (1990).

5. T. Sasaki, M. Kurita, T. Yabu, and T. Takahashi, Macromolecules, 28, 8528 (1995).

6. S. Yokotsuka, Y. Okada, Y. Tojo, T. Sasaki, and M. Yamamoto, Polym. J., 23, 95 (1991).

7. K. Ono, Y. Okada, S. Yokotsuka, S. Ito, and M. Yamamoto, Polym. J., 26, 199 (1994).

8. A. J. Kovacs and A. Gonthier, Kolloid Z. Z. Polymere, 250, 530 (1972).

9. S. Z. D. Cheng, J. Chen, J. S. Barley, A. Zhang, A. Habenschuss, and P. R. Zschack, Macromolecules, 25, 1453 (1992).

10. S. Z. D. Cheng, J. S. Barley, and P. A. Giusti, Polymer, 31, 845 (1990).

11. J. D. Hoffman and J. I. Lauritzen, J. Res. Natl. Bur. Stands., 65A, 297 (1961).

12. J. I. Lauritzen and J. D. Hoffman, J. Appl. Phys., 44, 4340 (1973).

13. J. I. Lauritzen, J. Appl. Phys., 44, 4353 (1973).

14. L. Mandelkern, J. Appl. Phys., 26, 443 (1955).

15. J. Boon and J. M. Azcue, J. Polym. Sci., A-2, 6, 885 (1968).

16. J. D. Hoffman and R. L. Miller, Polymer, 38, 3151 (1997).

17. D. Turnbull and J. C. Fisher, J. Chem. Phys., 17, 71 (1949).

18. E. Helfand, J. Chem. Phys., 54, 4651 (1971).

19. Y. Takahashi and H. Tadokoro, Macromolecules, 6, 672 (1973).

20. I. Bahar, B. Erman, and L. Monnerie, Macromolecules, 22, 2396 (1989), and papers cited therein.

21. H. D. Keith and F. J. Padden, J. Appl. Phys., 34, 2409 (1963). 